Research Article

\title{
Effect of Morphology Parameter Determination on Contact Behavior of Rock Joints under Compressive Loading
}

\author{
Feng Tian Tang and Zhi Cheng Tang \\ Faculty of Engineering, China University of Geosciences, Wuhan 430074, Hubei, China \\ Correspondence should be addressed to Zhi Cheng Tang; zctang@cug.edu.cn
}

Received 28 November 2020; Accepted 10 July 2021; Published 24 July 2021

Academic Editor: Hassan Nasir

Copyright (c) 2021 Feng Tian Tang and Zhi Cheng Tang. This is an open access article distributed under the Creative Commons Attribution License, which permits unrestricted use, distribution, and reproduction in any medium, provided the original work is properly cited.

\begin{abstract}
The closure behavior of rock joints is of critical importance to the study of hydromechanical behaviors and geophysical properties of jointed rock masses. Theoretical contact models, used to predict the relations of normal stress versus closure deformation, rely on morphology parameters of rock joint as the input parameters. The relevance of the contact models depends on the inherent assumptions and the accuracy with which the input parameters are determined. In the present study, morphology parameters of three rock joints are determined by the spectral moment approach and peak identification method, respectively. The differences are found to vary significantly depending on the selected method. The phenomenon would be related to the definition of an asperity peak on joint profile. The spectral method only considers the so-called asperity peaks, while the deterministic approach further accounts for the asperity shoulders. Finally, the morphology parameters determined by the two methods are treated as the input parameters of a validated theoretical model. The comparisons between the theoretical curves and the experimental results indicate that parameters determined by the deterministic method would be more reliable.
\end{abstract}

\section{Introduction}

Rock joints affect the mechanical properties of rocks, so do the morphology parameters of joints [1,2]. Topography of a solid surface has small-scale geometric features with random shapes and sizes, usually called "asperities." Distribution of asperities over the surface forms a random geometric structure which is in general known as "roughness," playing an important role in the mechanical behaviors of two contact rough surfaces, e.g., shear strength, closure deformation, or contact stiffness of rock joint. Various parameters have been proposed to describe rock joint topography based on different characterizing approaches. For rock joint, the application of available morphology parameters can fall broadly into three categories: (1) describing the geometrical features, such as Tse and Cruden [3], International Society for Rock Mechanics [4], Xie et al. [5], Belem et al. [6], Zhang et al. [7], and Li and Zhang [8]; (2) establishing the peak shear strength criteria, such as Barton and Choubey [9], Tatone and Grasselli [10], and Rasouli and Harrison [11]; and (3) serving as input parameters for theoretical contact models, such as Greenwood and Williamson [12], Brown and Scholz [13], Misra [14], Lanaro and Stephansson [15], and Xia et al. [16]. Here, we pay special attention to the last one, mainly including average peak radius of curvature $(\beta)$, peak density $(\eta)$, and standard deviation of peak height $(\sigma s)$. As such, the relevance of the theoretical models is dependent on their inherent assumptions and the accuracy with which the input parameters are determined.

Commonly, two methods are used to determine the mentioned morphology parameters. According to Nayak [17], a random and isotropic surface with a Gaussian height distribution can be adequately characterized by zeroth $\left(M_{0}\right)$, second $\left(M_{2}\right)$, and fourth $\left(M_{4}\right)$ moments of the power spectral density function. To better capture the topography of a natural solid surface with anisotropic roughness nature, average values of spectral moments obtained from a finite number of cross sections in a surface were used [18]. Essentially, the spectral moment approach is in a statistical sense [19], rather than a method based on the real asperities 
randomly distributed on the rough surface. The other method to determine the morphology parameters is based on individually identified asperity peaks as local maxima [20], and the above three parameters can then be calculated directly from these identified peaks, called as peak identification method (deterministic method). The deterministic method can avoid the averaging treatment inherent to the previously described spectral moment approach and is based on the actual 3D surface topography. Both the methods, suffering from varied sources of uncertainty, can serve as input parameters to theoretical models to describe the contact behaviors of a rock joint [21]. However, there is no analysis available in the literature to comprehensively compare the differences of the two methods for "real" rock joint (not numerically generated surfaces). Accordingly, the main objective of the present study is to provide a comprehensive understanding towards the quantification of morphology parameters of rock joints by the spectral moment approach and the deterministic method.

\section{Methodology}

2.1. Morphology of Rock Joint. The topography of a rock joint is usually composed of small-scale unevenness and largescale waviness [4], which have different influences on the joint closure behaviors $[16,21]$. Three rock joints with nominal length of $300 \mathrm{~mm}$ are selected for the analysis of peak spatial features, as shown in Figure 1. Rock joint J-I is composed of only unevenness component and J-II is composed of both unevenness and waviness components, while J-III is mainly composed of waviness component [22]. To sufficiently characterize the morphology, sample interval of $1.0 \mathrm{~mm}$ is used in $x$ and $y$ directions (generally, sample interval is within the range from 0.3 to $1.0 \mathrm{~mm}$ ). Average heights $(\mathrm{m})$ of the three rock joints are $1.65,3.58$, and $8.70 \mathrm{~mm}$, respectively. Gaussian distribution function, equation (1), is used to analyze the distribution of asperity height, and all the fitting results are listed in Table 1 [23]. The high correlation indicates that the morphologies of the three rock joints exhibit Gaussian distribution. Hence, the spectral moment approach can be used to evaluate the morphology parameters.

$$
f(z)=\frac{1}{\sqrt{2 \pi} \sigma} \exp \left[-\frac{1}{2}\left(\frac{z-\mu}{\sigma}\right)^{2}\right],
$$

where $f(\bullet)$ is the symbol of Gaussian function, $z$ is the surface height, $\sigma$ is the standard deviation of height, and $\mu$ is the expectation of height.

2.2. Spectral Moment Approach. For a given rough profile with a Gaussian height distribution, the asperity height denoted by $Z(x)$, the spectral moments, $M_{0}, M_{2}$, and $M_{4}$, can be determined by the following [17]:

$$
\begin{aligned}
& M_{0}=\operatorname{AVG}\left[\left(Z^{2}\right)\right], \\
& M_{2}=\operatorname{AVG}\left[\left(\frac{\mathrm{d} z}{\mathrm{~d} x}\right)^{2}\right], \\
& M_{4}=\operatorname{AVG}\left[\left(\frac{\mathrm{d}^{2} z}{\mathrm{~d} x^{2}}\right)^{2}\right],
\end{aligned}
$$

where AVG represents the arithmetic average.

Once $M_{0}, M_{2}$, and $M_{4}$ are known, the morphology parameters, $\beta, \eta$, and $\sigma s$, can then be calculated by [17]

$$
\begin{aligned}
\beta & =0.375 \sqrt{\frac{\pi}{m_{4}}}, \\
\eta & =\frac{1}{6 \pi \sqrt{3}}\left(\frac{m_{4}}{m_{2}}\right), \\
\sigma_{s} & =\left(1-\frac{0.8968}{\alpha}\right)^{0.5} \sqrt{m_{0}},
\end{aligned}
$$

where $\alpha=\left(m_{0} m_{4}\right) / m_{2}^{2}$, which is called the bandwidth parameter.

According to McCool [18], the value of the three parameters may vary significantly when calculated for an arbitrary single $2 \mathrm{D}$ trace. To overcome the limitations, 9 sectional profiles with equal spacing of $15 \mathrm{~mm}$ parallel to the analysis direction ( $x$ direction in the present study) are extracted (Figure 2). The spectral moments for each profile are calculated, and the averaged value is used to determine the corresponding morphology parameters.

2.3. Deterministic Method. As schematically shown in Figure 3, a peak on a profile is defined as a point with height higher than its $N$ adjacent points $(N=3,5,7, \ldots)$. An N-PP criterion identifies a point as a peak if its height exceeds that of its $(N-1)$ nearest neighbor points, resulting in $0.5(N-1)$ points on each side of the peak. Compared to 5PP and 7PP criteria, 3PP criterion would be more reliable to identify the peaks on a rock joint profile, which was also widely used [21, 22, 24-27]. The peak density and standard deviation of peak height can be directly calculated as all the peaks are identified. The radius of curvature for each peak is accurately and uniquely determined by its circumcircle. Then, the arithmetic average value of all the asperity peak radii is treated as the overall curvature of the profile.

\section{Analysis of Morphology Parameters}

3.1. Average Peak Radius of Curvature. Figure 4 shows the variations of the average peak radii of curvature for the three rock joints. We can clearly observe that with the increase of roughness (denoted by the average asperity height, similarly 


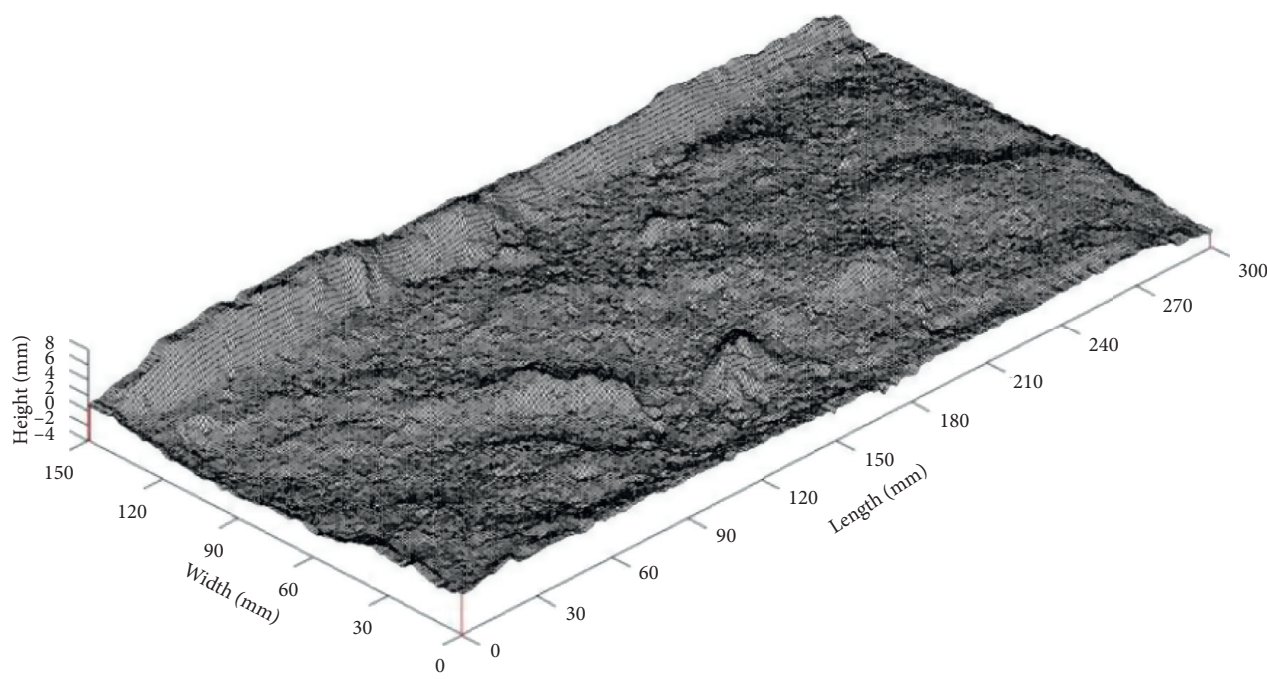

(a)

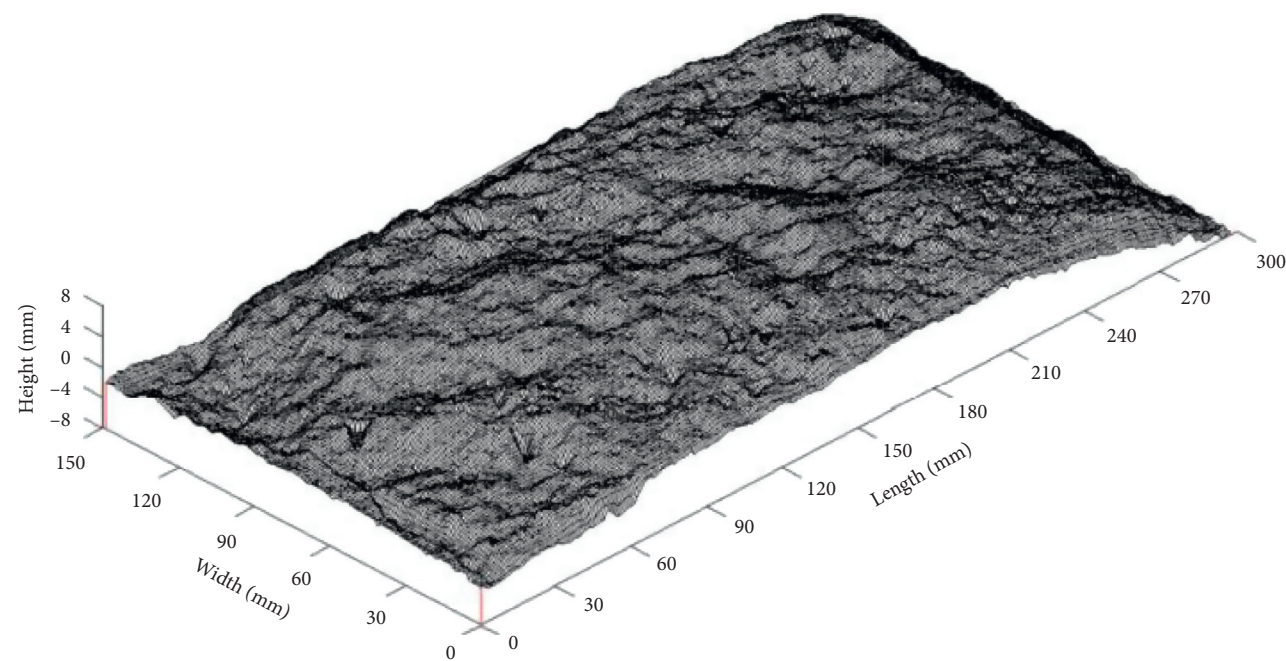

(b)

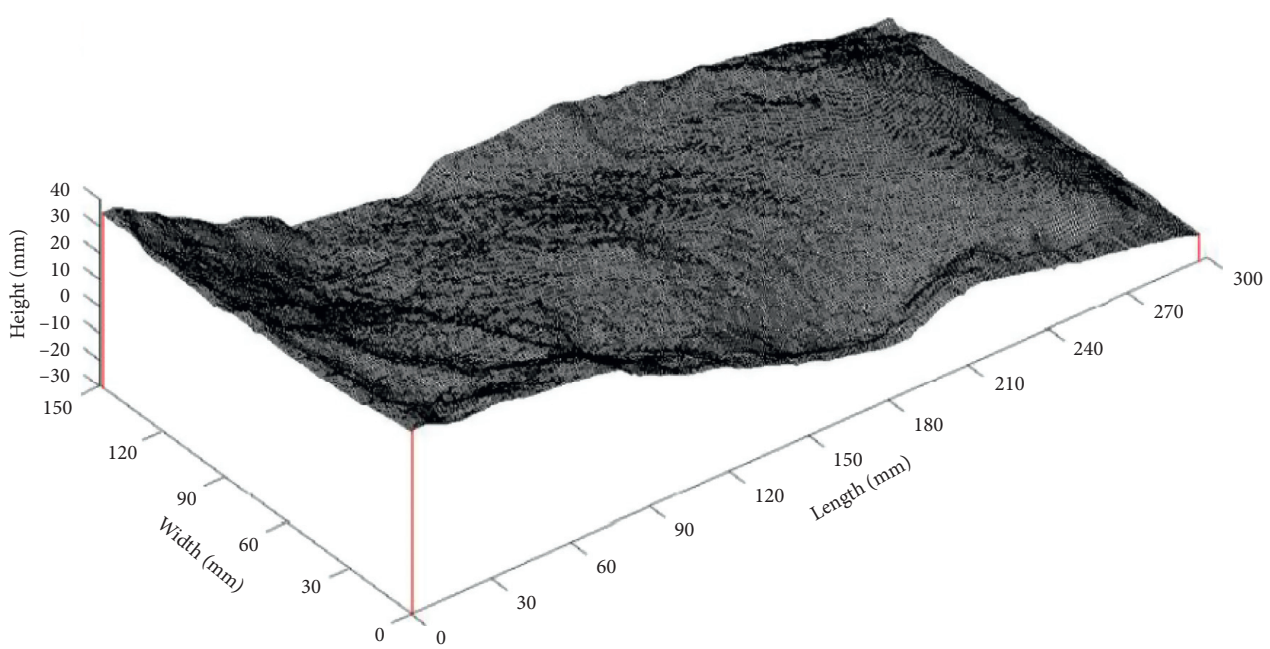

(c)

FIgURE 1: Morphology of the three rock joints. (a) J-I. (b) J-II. (c) J-III. 
TABle 1: Parameters for Gaussian distribution obtained by equation (1).

\begin{tabular}{lccc}
\hline Sample & \multicolumn{2}{c}{ Fitting parameters } & Correlation coefficient \\
\hline J-I & $\mu$ & 2.21 & 0.994 \\
J-II & 0.33 & 1.86 & 0.928 \\
J-III & 1.12 & 1.57 & 0.909 \\
\hline
\end{tabular}

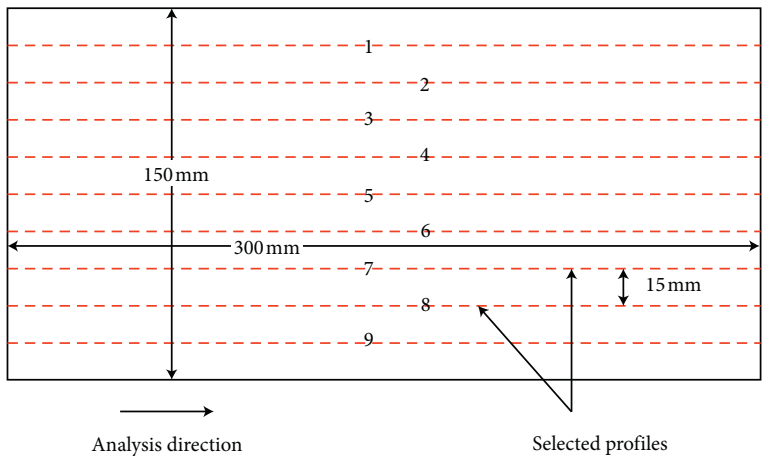

(a)

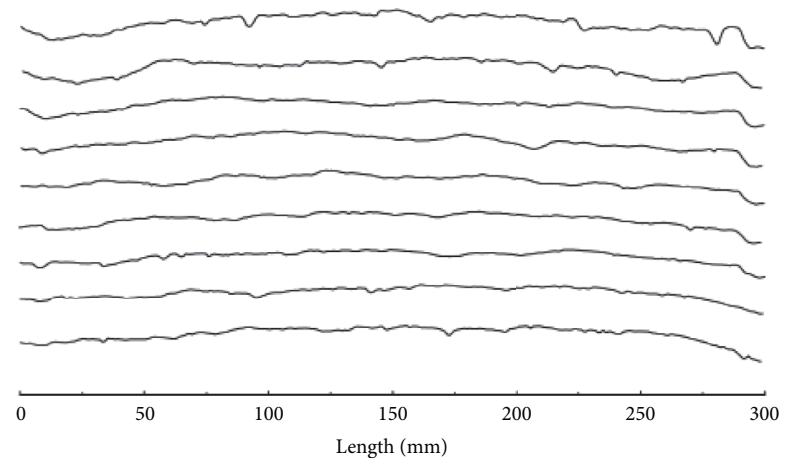

(b)

Figure 2: Method to select joint profiles. (a) Position of joint profiles for morphology analysis. (b) Selected joint profiles (J-II is taken as an example).

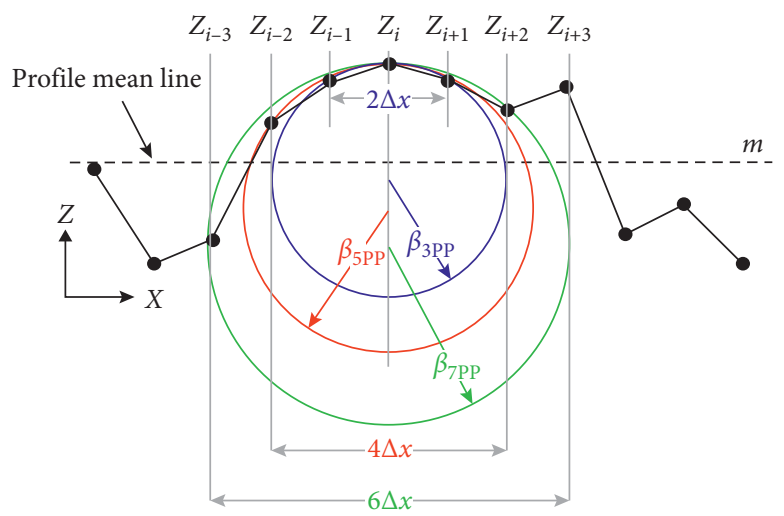

FIGURE 3: Schematic plot for 3PP, 5PP, and 7PP criteria on rock joint profile.

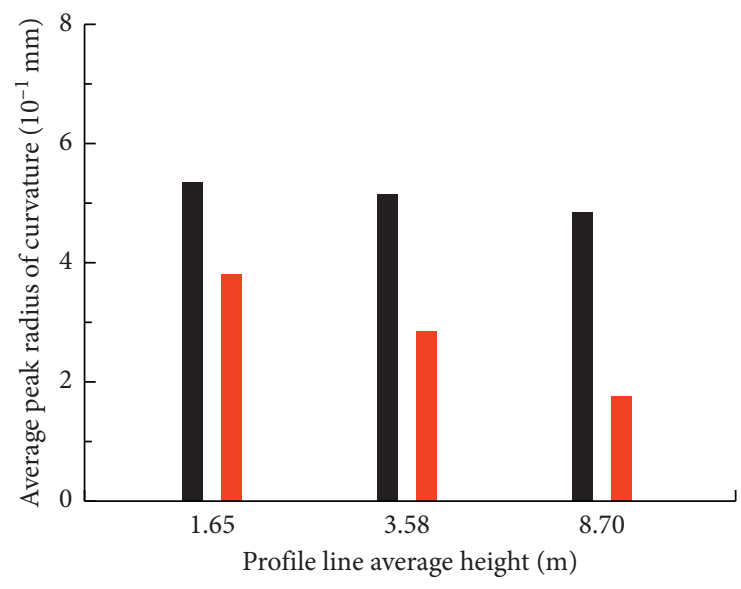

Peak identification method Spectral moment approach

FIGURE 4: Average peak radius of curvature for the three joint profiles. 
hereinafter), the radii determined by the peak identification method exhibit small volatility, while the ones determined by the spectral moment approach are significantly lower and exhibit an obvious downward trend throughout the whole roughness range. As the average asperity heights increase from 1.65 to $8.70 \mathrm{~mm}$, the average peak radii of curvature determined by the spectral moment approach decrease from 0.386 to $0.182 \mathrm{~mm}$, with a reduction of about $52.8 \%$, while the ones determined by the peak identification method decrease from 0.55 to $0.44 \mathrm{~mm}$, only with a reduction of about $18.5 \%$. With consideration of the nature of the two methods, it can be inferred that the spectral moment approach would underestimate the average peak radius of curvature and there is a more pronounced trend as surface roughness increases.

3.2. Peak Density. Figure 5 shows the variations of peak density for the three rock joints. In the present study, the peak density means the linear density that is calculated by the peak number divided by the profile nominal length. The peak density determined by the peak identification method decreases with the increase of roughness. However, there is an onward trend throughout the whole roughness range for the one determined by the spectral moment approach. As the average asperity heights increase, the peak density determined by the spectral moment approach increases from 0.39 to 0.46 , with an increase of about $17.9 \%$, while the one determined by the peak identification method decreases from 0.28 to 0.15 , with a reduction of about $46.4 \%$. The peak density is also reported to decrease with the increase of roughness, as is the case for the peak identification method which is in line with many theoretical and experimental observations for rough surfaces $[28,29]$. As such, it can be inferred that the results for the peak identification method have a much more trustworthy physical background than the ones for the spectral moment approach. Essentially, the deterministic method imposes a stricter definition of an asperity peak and results in a lower peak density, while the spectral moment approach does not necessarily trace the real asperity peaks, but possibly the shoulder of an asperity [19], thus resulting in a higher peak density.

3.3. Standard Deviation of Peak Height. Figure 6 shows the variations of the standard deviation of peak heights for the three rock joints with varied roughness. The peak identification method always results in a higher standard deviation throughout the roughness range. The absolute difference and the ratio, $\sigma_{s \_ \text {SMA }} / \sigma_{s \_ \text {PIM }}$, increase as the roughness increases, where $\sigma_{s_{-} \text {SMA }}$ and $\sigma_{s_{-} \text {PIM }}$ are the values determined by the spectral moment approach and peak identification method, respectively. For the spectral moment approach, the standard deviation of peak height increases almost linearly with the increase of roughness $\left(R^{2}=0.89\right)$, while the other shows an exponential relation $\left(R^{2}=0.96\right)$. The peak identification method accounts for the strictly determined asperity peaks on a profile (Figure 3), whereas the spectral moment approach may rely on peak shoulders, rather than the real peaks, resulting in a lower standard deviation of asperity peak heights.

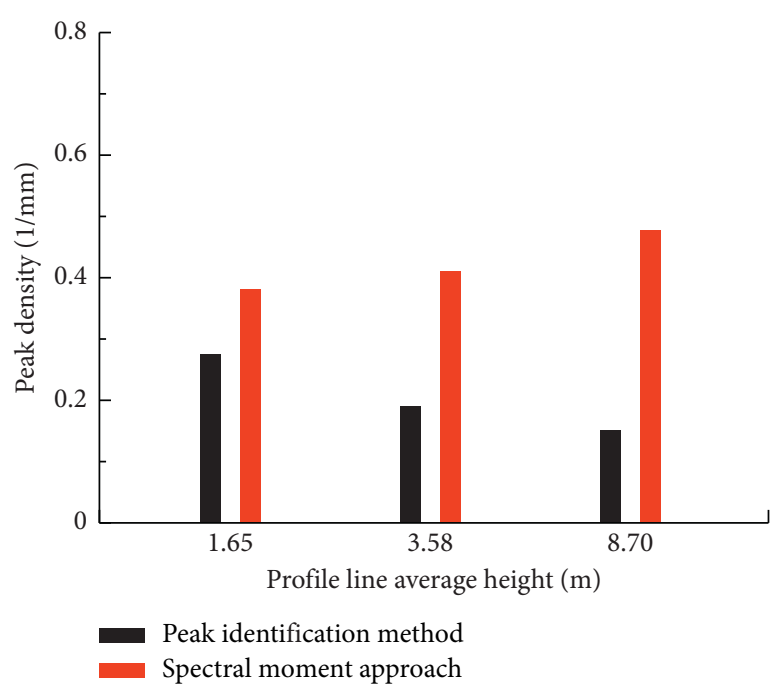

Figure 5: Peak density for the three joint profiles.

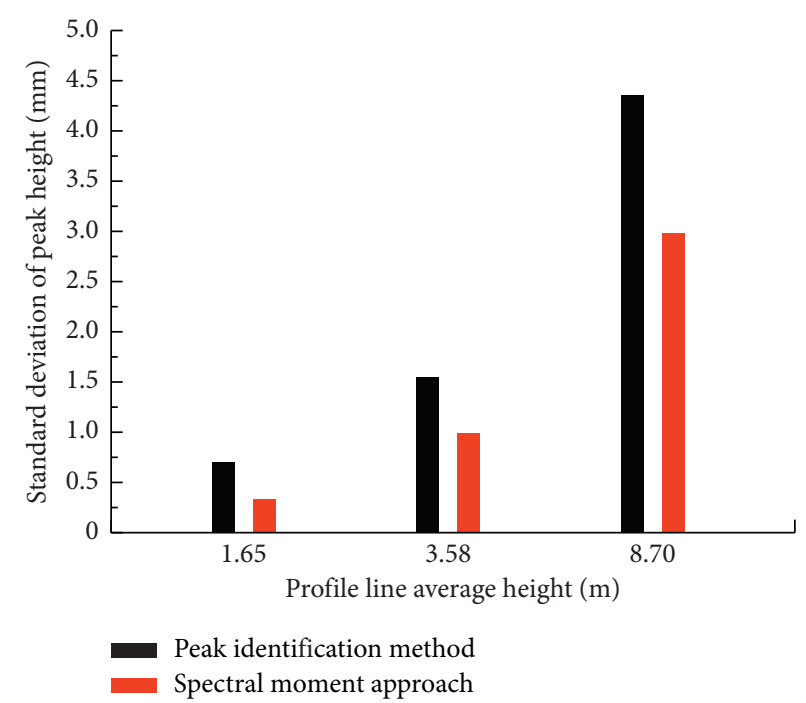

FIgURE 6: Standard deviation of peak height for the three joint profiles.

\section{Theoretical Application}

In this section, the effectiveness of determining the morphology parameters is illustrated by comparing predictions by an available theoretical closure model with experimental results.

4.1. Theoretical Model. Recently, a theoretical closure model with the inclusion of asperity interaction was developed by the first author and his co-workers [27], which can partly capture the closure behaviors between a "waviness" surface and an "unevenness" surface based on static equilibrium and deformation compatibility. As shown in Figure 7, the waviness is simplified by a periodic function and has identical amplitude. When an external load $P$ is applied to a waviness surface, the pressure distribution of contact area for waviness $(r)$ can be determined by equation (4). Then, the 


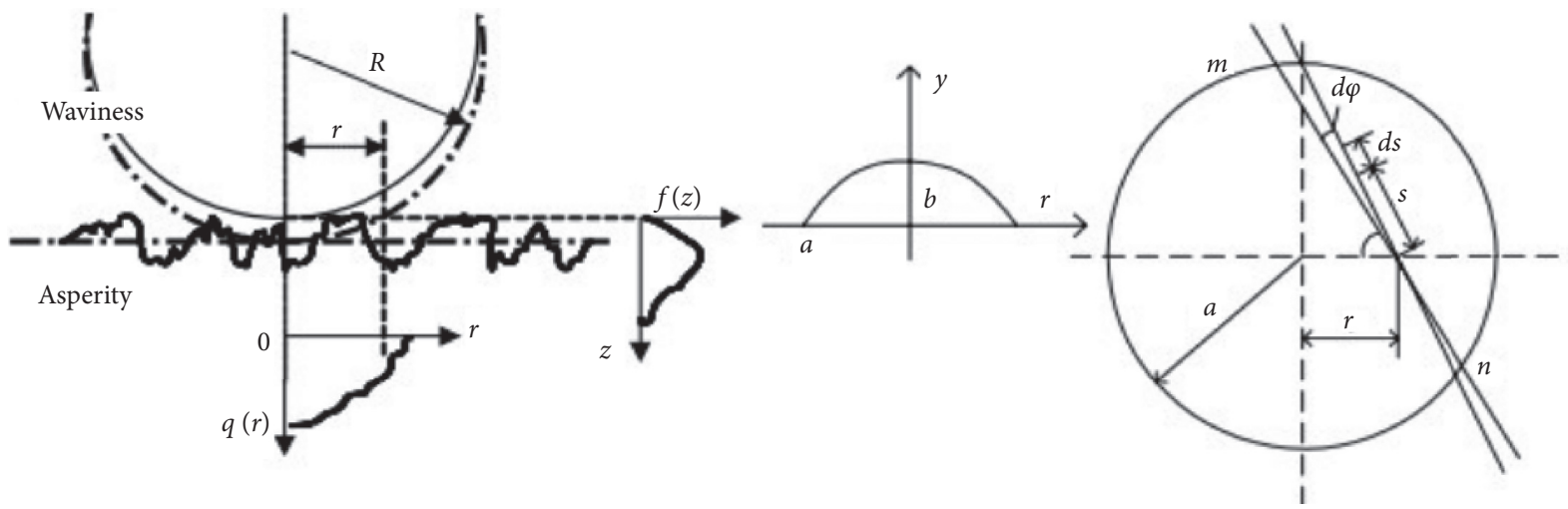

FIgURE 7: Contact between a "waviness" and an "unevenness."

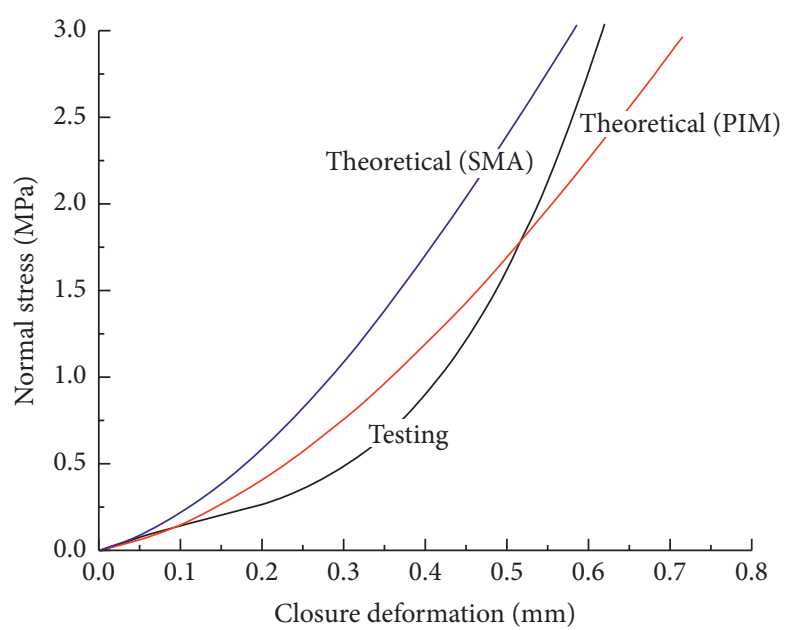

(a)

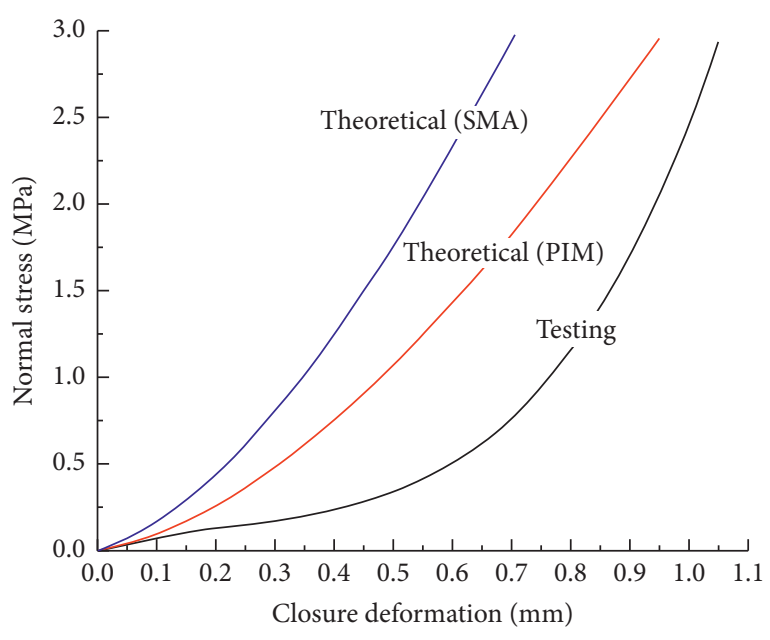

(b)

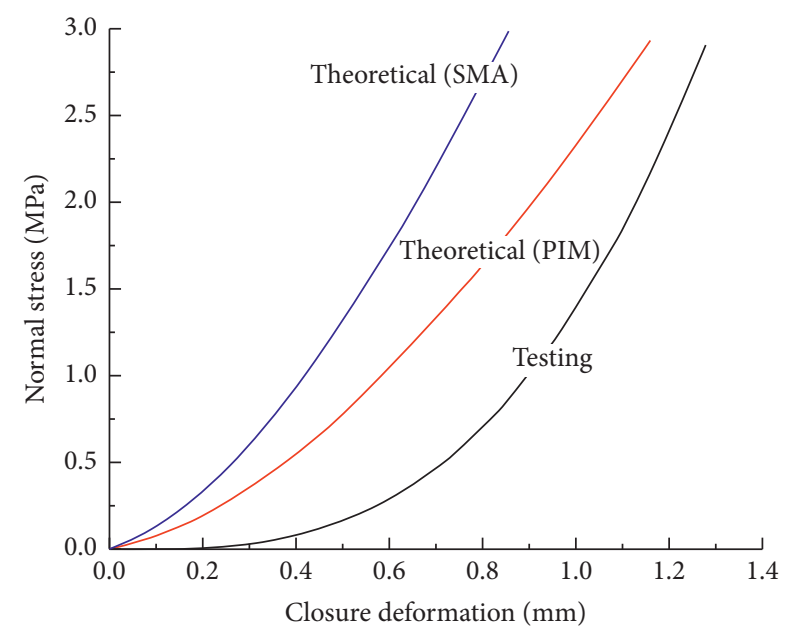

(c)

FIgURE 8: Comparison between experimental results and calculated curve. (a) J-I. (b) J-II. (c) J-III. 
waviness deformation $(r)$ and the asperity deformation $(r)$

can be determined by equations (5) and (6), respectively.

$$
\begin{aligned}
q_{w}(r) & =\frac{p}{\pi c}\left(\frac{1}{r^{2}+4 b^{2}}-\frac{1}{a^{2}+4 b^{2}}\right), \\
w(r) & =\frac{2 P}{\pi^{2} E^{\prime} b c} \int_{0}^{\pi / 2}\left[a \tan \left(\frac{1}{2 b} \sqrt{a^{2}-r^{2} \sin ^{2} \varphi}\right)-\frac{2 b}{a^{2}+4 b^{2}} \sqrt{a^{2}-r^{2} \sin ^{2} \varphi}\right] \mathrm{d} \varphi, \\
u(r) & =\frac{a^{2}-r^{2}}{2 R}+w(a)-w(r),
\end{aligned}
$$

where $r$ is the distance from the contact center; $R$ is the curvature radius of waviness; $a, b$ are the radii of circular contact area and a special curve, respectively; $\varphi$ is the angle shown in Figure 7; and $c=\ln \left(a^{2}+4 b^{2} / 4 b^{2}\right)-a^{2} / a^{2}+4 b^{2}$.

The effect of deformed asperity interaction on the overall closure deformation of a rock joint is considered by a simplified approach as a uniform displacement on the surface caused by a mean pressure, $p_{\text {nom, }}$ which can be directly estimated by the Greenwood and Williamson model [8] by the first iteration. As the pressure distribution of asperity contact is relevant to the asperity deformation $(r)$, the asperity pressure $(r)$ at the contact area can be given by the following equation:

$$
q_{a}(r)=\frac{4}{3} \eta E^{\prime} \sqrt{B} \int_{0}^{u(r)}\left[u(r)+p_{\text {nom }} \frac{\sqrt{A}}{E \prime-z}\right]^{3 / 2} f(z) \mathrm{d} z,
$$

where $E^{\prime}=E / 2\left(1-v^{2}\right)$ in which $E$ is Young's modulus and $v$ is Poisson's ratio; $A$ is the nominal contact area.

The steps to solve the model are as follows: (1) assign non-zero initial values for $a, b$ and calculate the waviness deformation, $w(r)$ and $w(a)$, by equation (5); (2) calculate the asperity deformation, $u(r)$, by equation (6); (3) calculate the contact force by using equation (7) on the basis of the asperity deformation determined by equation (6); and (4) repeat the above procedures until the following equilibrium conditions are satisfied: $q_{a}(0)=q_{w}(0)$ and $q_{a}(a / 2)=q_{w}$ $(a / 2)$.

4.2. Comparisons. Tang et al. [22] and Tang [23] performed a series of closure tests on artificial rock joints with Young's modulus $E=6.1 \mathrm{GPa}$ and Poisson's ratio $v=0.16$. The morphologies of the three rock joints are shown in Figure 1. Figure 8 illustrates the comparison between experimental and theoretical results for the three joints. Generally, the theoretical curves solved by the model with morphology parameters determined by the peak identification method are closer to the experimental data than the case of the spectral moment approach. Thus, it seems to be more reliable to use the peak identification method to analyze the contact behaviors of rock joint as it includes all available information of the surface rather than relying on a statistical sense. However, it should be noted that the contact spots are three-dimensional for any real contact between rough rock joints, leading to the discrepancy between the testing curve and theoretical curve.

\section{Conclusions}

Due to the fact that the relevance of theoretical contact models is dependent on the peak identification assumptions and the determination accuracy, the present study provides a comparison for determining the input parameters of these theoretical models by using two methods. The peak identification method follows a stricter definition of an asperity peak on a surface and can avoid the statistical averaging, while the spectral moment approach is based on $2 \mathrm{D}$ traces that may probably trace peak shoulders rather than the peaks. As such, a larger peak density and a lower standard deviation of peak height can be observed for the spectral moment approach. In addition, the spectral moment approach underestimates the average peak radius of curvature for the three rock joint profiles, which would be more pronounced as the roughness increases. The effectiveness of determining morphology parameters is verified by comparing the curves determined by a theoretical model with the experimental results. It would be more reliable to use the morphology parameters obtained by the deterministic method to predict the contact behaviors of rock joint.

\section{Data Availability}

The data used to support the findings of this study are included within the article.

\section{Conflicts of Interest}

The authors declare that there are no conflicts of interest regarding the publication of this paper.

\section{Acknowledgments}

The authors gratefully acknowledge the support from the National Natural Science Foundation of China under project no. 41672302 and the Fundamental Research Funds for the Central Universities, China University of Geosciences (Wuhan), under project no. CUGGC09. 


\section{References}

[1] Y. Liu, F. Dai, L. Dong, N. Xu, and P. Feng, "Experimental investigation on the fatigue mechanical properties of intermittently jointed rock models under cyclic uniaxial compression with different loading parameters," Rock Mechanics and Rock Engineering, vol. 51, no. 1, pp. 47-68, 2018.

[2] Y. Liu, F. Dai, P. Fan, N. Xu, and L. Dong, "Experimental investigation of the influence of joint geometric configurations on the mechanical properties of intermittent jointed rock models under cyclic uniaxial compression," Rock Mechanics and Rock Engineering, vol. 50, no. 6, pp. 1453-1471, 2017.

[3] R. Tse and D. M. Cruden, "Estimating joint roughness coefficients," International Journal of Rock Mechanics and Mining Science \& Geomechanics Abstracts, vol. 16, no. 5, pp. 303-307, 1979.

[4] International Society for Rock Mechanics, "Suggested methods for the quantitative description of discontinuities in rock masses," International Journal of Rock Mechanics and Mining Science \& Geomechanics Abstracts, vol. 15, no. 6, pp. 319-368, 1978.

[5] H. Xie, J.-A. Wang, and M. A. Kwaśniewski, "Multifractal characterization of rock fracture surfaces," International Journal of Rock Mechanics and Mining Sciences, vol. 36, no. 1, pp. 19-27, 1999.

[6] T. Belem, M. Souley, and F. Homand, "Modeling surface roughness degradation of rock joint wall during monotonic and cyclic shearing," Acta Geotechnica, vol. 2, no. 4, pp. 227-248, 2007.

[7] G. Zhang, M. Karakus, H. Tang, Y. Ge, and L. Zhang, "A new method estimating the $2 \mathrm{D}$ joint roughness coefficient for discontinuity surfaces in rock masses," International Journal of Rock Mechanics and Mining Sciences, vol. 72, pp. 191-198, 2014.

[8] Y. Li and Y. Zhang, "Quantitative estimation of joint roughness coefficient using statistical parameters," International Journal of Rock Mechanics and Mining Sciences, vol. 77, pp. 27-35, 2015.

[9] N. Barton and V. Choubey, "The shear strength of rock joints in theory and practice," Rock Mechanics and Rock Engineering, vol. 10, no. 1, pp. 1-54, 1977.

[10] B. S. Tatone and G. Grasselli, "A method to evaluate the threedimensional roughness of fracture surfaces in brittle geomaterials," Review of Scientific Instruments, vol. 80, no. 12, Article ID 125110, 2009.

[11] V. Rasouli and J. P. Harrison, "Assessment of rock fracture surface roughness using Riemannian statistics of linear profiles," International Journal of Rock Mechanics and Mining Sciences, vol. 47, no. 6, pp. 940-948, 2010.

[12] J. A. Greenwood and J. B. P. Williamson, "Contact of nominally flat surfaces," Proceedings of the Royal Society of London-Series A: Mathematical and Physical Sciences, vol. 295, no. 1442 , pp. 300-319, 1966.

[13] S. R. Brown and C. H. Scholz, "Closure of random elastic surfaces in contact," Journal of Geophysical Research: Solid Earth, vol. 90, no. B7, pp. 5531-5545, 1985.

[14] A. Misra, "Micromechanical model for anisotropic rock joints," Journal of Geophysical Research: Solid Earth, vol. 104, no. B10, pp. 23175-23187, 1999.

[15] F. Lanaro and O. Stephansson, "A unified model for characterisation and mechanical behaviour of rock fractures," Thermo-Hydro-Mechanical Coupling in Fractured Rock, vol. 160, no. 5, pp. 989-998, 2003.
[16] C. C. Xia, Z. Q. Yue, L. G. Tham, C. F. Lee, and Z. Q. Sun, "Quantifying topography and closure deformation of rock joints," International Journal of Rock Mechanics and Mining Sciences, vol. 40, no. 2, pp. 197-220, 2003.

[17] P. R. Nayak, "Random process model of rough surfaces," Journal of Lubrication Technology, vol. 93, no. 3, pp. 398-407, 1971.

[18] J. I. Mc Cool, "Relating profile instrument measurements to the functional performance of rough surfaces," Journal of Tribology, vol. 109, no. 2, pp. 264-270, 1987.

[19] B. Bhushan and P. L. Ko, "Introduction to tribology," Applied Mechanics Reviews, vol. 56, no. 1, pp. B6-B7, 2003.

[20] J. A. Greenwood, "A unified theory of surface roughness," Proceedings of the Royal Society of London. A. Mathematical and Physical Sciences, vol. 393, no. 1804, pp. 133-157, 1984.

[21] Z. C. Tang, Y. Y. Jiao, and L. N. Y. Wong, "Theoretical model with multi-asperity interaction for the closure behavior of rock joint," International Journal of Rock Mechanics and Mining Sciences, vol. 97, pp. 15-23, 2017.

[22] Z.-C. Tang, Q.-S. Liu, C.-C. Xia, Y.-L. Song, J.-H. Huang, and C.-B. Wang, "Mechanical model for predicting closure behavior of rock joints under normal stress," Rock Mechanics and Rock Engineering, vol. 47, no. 6, pp. 2287-2298, 2014.

[23] Z.-C. Tang, Mechanical behaviors of rock joint under different contact state and columnar jointed rock mass, Ph.D thesis, Tongji University, Shanghai, China, 2013.

[24] G. Swan, "Determination of stiffness and other joint properties from roughness measurements," Rock Mechanics and Rock Engineering, vol. 16, no. 1, pp. 19-38, 1983.

[25] G. Swan and S. Zongqi, "Prediction of shear behaviour of joints using profiles," Rock Mechanics and Rock Engineering, vol. 18, no. 3, pp. 183-212, 1985.

[26] Z. C. Tang and Y. Y. Jiao, "A self-consistent model with asperity interaction for the mechanical behavior of rock joints under compressive loading," International Journal of Rock Mechanics and Mining Sciences, vol. 100, pp. 23-27, 2017.

[27] C. Y. Poon and B. Bhushan, "Surface roughness analysis of glass-ceramic substrates and finished magnetic disks, and $\mathrm{Ni}$ P coated Al-Mg and glass substrates," Wear, vol. 190, no. 1, pp. 89-109, 1995.

[28] Z. C. Tang, C. C. Xia, Y. Y. Jiao, and L. N. Y. Wong, “Closure model with asperity interaction in normal contact for rock joint," International Journal of Rock Mechanics and Mining Sciences, vol. 83, pp. 170-173, 2016.

[29] G. Zavarise, M. Borri-Brunetto, and M. Paggi, "On the reliability of microscopical contact models," Wear, vol. 257, no. 3, pp. 229-245, 2004. 